\title{
Characterization of a monoclonal antibody directed against a sulphoglycolipid that is evolutionarily conserved and developmentally regulated in rat brain
}

\author{
E. Borroni, E.A. Derrington, and V.P. Whittaker \\ Arbeitsgruppe Neurochemie, Max-Planck-Institut für biophysikalische Chemie, Göttingen, Federal Republic of Germany
}

\begin{abstract}
Summary. Monoclonal antibodies (MABs) have been raised against acidic glycolipids extracted from the electric organ of Torpedo marmorata. One of these, designated L9, appears to recognize acidic glycolipids in adult $T$. marmorata electric organ, electromotor nerves and brain, adult rat sciatic nerve, and in embryonic and neonatal rat brain, starting at embryonic day (ED) 15 and disappearing by the 20th day of post-natal life. The epitope is present in growth cones isolated from 4-day-old rats; its proportion relative to total gangliosides is, however, no higher than that found in whole neonatal brain membranes. Desialidation of the acidic glycolipid fraction modifies neither the immunoreactivity nor the $R_{F}$ value following thin-layer chromatography (TLC) of the antigen; it is concluded that the antigen is not a ganglioside. The MAB, HNK-1, recognizes the $\mathrm{L} 9$ antigen. Both $\mathrm{HNK}-1$ and $\mathrm{L} 9$ recognize a sulphoglycolipid of the same $R_{F}$ in TLC. The function of the L9 antigen is not known but its evolutionary conservation, presence in growth cones and its developmental regulation in the mammalian central nervous system indicate that it plays an important role in nervous system maturation.
\end{abstract}

Key words: Sulphoglycolipids - Developmental regulation HNK-1 (monoclonal antibody) - Torpedo marmorata (Elasmobranchii)

The discovery that certain glycolipids of the ganglioside class, designated Chol-1, act as transmitter-specific surface markers for cholinergic nerve terminals (Richardson et al. 1982; Ferretti and Borroni 1986) has prompted our interest in a possible role of acidic glycolipids in general as surface molecules involved in synapse formation and maturation (Ferretti and Borroni 1984; Fiedler et al. 1986). We have therefore utilized the monoclonal antibody (MAB) technique in conjunction with a purely cholinergic preparation of presynaptic plasma membranes derived from the electromotor nerve terminals of Torpedo marmorata to characterize such acidic glycolipids more fully. In the course of this work, a MAB designated L9 has been obtained; this recognizes a glycolipid which, though different from Chol-1, has nevertheless proved to have interesting features. The L9 glycolipid is expressed in the central and peripheral nervous

Send offprint requests to: Dr. E. Borroni, AG Neurochemie, MaxPlanck-Institut für biophysikalische Chemic, Postfach 2841, D3400 Göttingen, Federal Republic of Germany system of embryonic and adult $T$. marmorata but, interestingly, the expression of the antigen appears to be under strict developmental regulation in the brain of mammals. Furthermore, the L9 glycolipid is also recognized by the MAB, HNK-1, which has originally been raised against a plasma membrane antigen from a human T-cell line, HSB2 (Abo and Blach 1981; Abo et al. 1982). The HNK-1 epitope has been shown to be present in various other glycoproteins, many of which are known to be involved in adhesive phenomena among neural cells. Among these are the neural cell adhesion molecules N-CAM (Kruse et al. 1984), cytotactin (Grumet et al. 1985), L1, L2, and J1 (Rathjen and Schachner 1984; Kruse et al. 1985), and the myelinassociated glycoprotein (Poltorak et al. 1987). In fish, the epitope has been shown to be present in a group of glycoproteins, called ependymins, that are secreted into the cerebrospinal fluid of goldfish during learning (Shashoua et al. 1986) and in a subset of acetylcholinesterase molecules present in the electric organ of Electrophorus and Torpedo spp. (Bon et al. 1987). Furthermore, antibodies to the HNK-1 epitope have been shown to be produced in certain human polyneuropathies (Ilyas et al. 1984a, b), suggesting an involvement of antigens bearing this epitope in neuropatho$\log y$.

In the work presented here we describe the developmental expression of the L 9 glycolipid and its subcellular distribution. In addition, in view of the similarity of the MAB L9 and HNK-1, we have carried out a careful comparison of the two MABs. Preliminary accounts of this work have been given (Borroni and Derrington 1987; Borroni et al. 1987).

\section{Materials and methods}

\section{Materials}

Ganglioside standards were obtained from Seromed (Munich, FRG). Plates for thin-layer chromatography (TLC) were from Merck (Darmstadt, FRG).

\section{Animals}

Specimens of Torpedo marmorata were obtained from the Station Biologique d'Arcachon, France, and were maintained in an aquarium in the laboratory at $15-17^{\circ} \mathrm{C}$ until used.

Non-pregnant, pregnant and 4-day-old rats were of the 
Wistar strain and were from the Institute's breeding colony. Embryonic day (ED) 0 was determined by the presence of sperm in the intravaginal lavage of breeding females.

$\mathrm{Balb} / \mathrm{c}$ mice were obtained commercially as required.

\section{Tissues, tissue fractions and extracts}

Tissues. Electric organ, electromotor nerves and brain of T. marmorata were removed from fish anaesthetized by immersion in artificial sea water containing $0.05 \%$ of Tricaine methane sulphonate (Sigma, Munich, FRG).

Embryonic rat brains were removed from the embryos under a dissecting microscope. Sciatic nerves were obtained from adult rats and forebrain cortical tissue from 4-day-old rats.

Tissues destined for acidic glycolipid extraction were frozen in liquid nitrogen immediately after dissection and stored at $-70^{\circ} \mathrm{C}$ until used.

Rat cortical membranes. Cortical tissue from 4-day-old rats was homogenized in 9 volumes of $0.32 \mathrm{M}$ sucrose, $4 \mathrm{mM}$ HEPES-NaOH buffer, $\mathrm{pH} 7.4$ in a motor-driven Teflon and glass homogenizer with 6 up-and-down strokes at $840 \mathrm{rev}$. $\min ^{-1}$. The homogenate was layered onto $1.2 \mathrm{M}$ sucrose in the tubes of a Beckman Ti-70 fixed-angle rotor and centrifuged at $45000 \mathrm{rev} \cdot \mathrm{min}^{-1}$ for $15 \mathrm{~min}$. The material collecting at the sample $1.2 \mathrm{M}$ sucrose interface was centrifuged into $0.4 \mathrm{M}$ sucrose under the same conditions.

Growth cones. A fraction enriched in growth cones was prepared from cortical tissue from 4-day-old rats by a slight modification of the method of Gordon-Weeks and Lockerbie (1984).

Tissue fractions were frozen and stored as for tissue samples.

Extraction of tissues and tissue fractions. Ganglioside fractions were extracted from tissues and tissue fractions using the method of Tettamanti et al. (1973). Although these fractions are highly enriched in gangliosides they also contain other acidic glycolipids. We therefore refer to these fractions as acidic glycolipids. Desialidation of extracts was achived by digestion with neuraminidase as previously described (Ferretti and Borroni 1986). The completion of the reaction was controlled by TLC.

Cell culture. Spinal cords were carefully dissected from mouse embryos (ED 13) under a dissection microscope. After trituration with sharp scissors the spinal cord fragments were incubated at $37^{\circ} \mathrm{C}$ for $20 \mathrm{~min}$ in $0.125 \%$ trypsin, $0.5 \%$ DNase in calcium/magnesium-free Earle's medium. Dissociated cells were plated on glass coverslips coated with collagen and grown in $10 \%$ foetal calf serum, $10 \%$ horse serum in Dulbecco's Modified Eagle Medium.

\section{Immunochemical techniques}

Preparation and screening of MABs. Balb/c mice were immunized with acidic glycolipids from the electric organ of Torpedo marmorata according to Galanos et al. (1971) except that delipidated $E$. coli were used as the adjuvant instead of Salmonella. MABs were screened for their ability to immunostain glycolipids separated by TLC according to the method of Brockaus et al. (1981) as modified by
Ferretti and Borroni (1986). The immune-stained bands were quantified by scanning in a Shimadzu C5-930 Dual Wavelength TLC scanner as described by Derrington et al. (1988).

Both L9 and HNK-1 MABs were of the IgM type and were compared at equal IgM concentrations; the IgM contents were measured using the enzyme-linked immunosorbent assay (ELISA)-sandwich technique employing rabbit anti-mouse IgM specific for the $F_{c}$ fragment of IgM immobilized in the wells and affinity-purified, peroxidase-conjugated goat anti-mouse IgM.

Immunohistochemistry. Rats were anaesthetized with sodium pentobarbital and transcardially perfused with $250 \mathrm{ml}$ of $0.15 \mathrm{M} \mathrm{NaCl}-50 \mathrm{mM}$ sodium phosphate buffer, $\mathrm{pH} 7.4$ (phosphate-buffered saline, PBS), followed by $250 \mathrm{ml}$ of $4 \%$ paraformaldehyde in $0.1 \mathrm{M}$ phosphate buffer, $\mathrm{pH}$ 7.4. Brain and spinal cord were dissected out and immersionfixed in $4 \%$ paraformaldehyde for another $2-4 \mathrm{~h}$. Sections (30 $\mu \mathrm{m}$ thick) were cut using an Oxford Vibratome. After a brief rinsing in $0.15 \mathrm{M} \mathrm{NaCl}-50 \mathrm{mM}$ Tris- $\mathrm{HCl}$ buffer, $\mathrm{pH} 7.4$ (Tris-buffered saline, TBS) and preincubation in TBS containing $5 \%$ normal rabbit serum and $0.2 \%$ Triton $\mathrm{X}-100$ for $1 \mathrm{~h}$, sections were incubated with the MAB, L9, or with MAB, HNK-1, diluted 1:5 in 5\% normal rabbit serum for $12-14 \mathrm{~h}$ at $4^{\circ} \mathrm{C}$. After extensive washing with TBS, sections were incubated for $2 \mathrm{~h}$ with biotin-conjugated rabbit anti-mouse immunoglobulins (DAKO, Hamburg, FRG) diluted 1:1000 in 5\% normal rabbit serum. After another cycle of washing, the sections were incubated for 30 min with the $\mathrm{ABC}$ complex (DAKO, Hamburg; the complex was formed by mixing avidin and biotinylated horseradish peroxidase according to the instruction of the

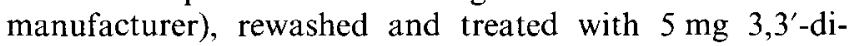
amino-benzidine and $3.3 \mu \mathrm{l}$ of $30 \% \mathrm{H}_{2} \mathrm{O}_{2}$ in $10 \mathrm{ml}$ TBS. Finally, the sections were dehydrated and mounted with Eukitt. Cells grown in culture were fixed for $30 \mathrm{~min}$ in $4 \%$ paraformaldehyde in $0.1 \mathrm{M}$ phosphate buffer, $\mathrm{pH}$ 7.4. After $1 \mathrm{~h}$ preincubation in $10 \%$ normal goat serum in PBS, the cells were incubated with MAB, L9. After a cycle of washing, the cells were incubated for $1 \mathrm{~h}$ in rhodamine-conjugated goat anti-mouse IgM (Paesel, Frankfurt, FRG) diluted $1: 200$ in $10 \%$ normal goat serum.

In some experiments, two sets of cells, fixed and not fixed respectively, were incubated for $30 \mathrm{~min}$ in cold $\left(-20^{\circ} \mathrm{C}\right)$ chloroform/methanol 2:1 $(\mathrm{v} / \mathrm{v})$ in order to extract lipidic components. After this incubation, cells were fixed with paraformaldehyde and processed for immunohistochemistry as described above.

\section{Results}

$M A B L 9$ recognizes an acidic glycolipid which is developmentally regulated in the rat

$M A B$ s raised against T. marmorata glycolipids. Colonies of hybridoma were obtained by fusing the spleen cells of a mouse immunized with a fraction of acidic glycolipid prepared from the electric organ of T. marmorata. Of the 369 colonies obtained, 96 produced antibodies directed against electric organ glycolipids and were further screened against crude rat brain glycolipids for possible cross-reactivity. There were 37 positive colonies, one of which was found to recognize a developmentally regulated glycolipid (see be- 


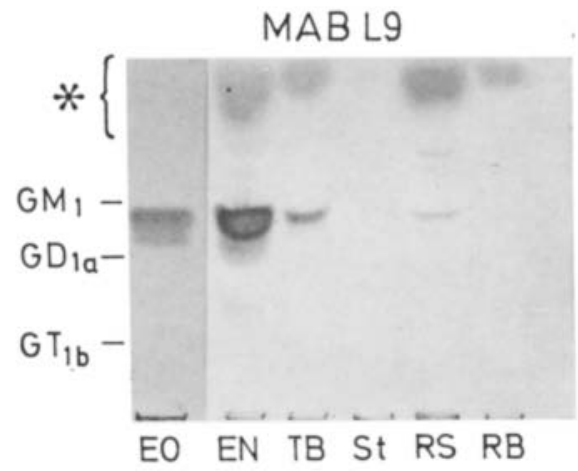

Fig. 1. Reactivity of MAB L9 against glycolipids extracted from T. marmorata and rat nervous systems. Acidic glycolipid extracted from $T$. marmorata electric organ $(E O)$, electric nerves $(E N)$ and brain $(T B)$, and from adult rat sciatic nerve $(R S)$ and brain $(R B)$ were separated on TLC and immune stained with MAB L9 or sprayed with Ehrlich's reagent to render the total glycolipid pattern visible. An immunoreactive band migrating between the standard gangliosides GMI and GD1a is detected by $M A B L 9$ in all the glycolipidic fractions with the exception of rat brain. The staining observed in the area indicated by the asterisk should be considered as non-specific since an identical pattern of staining can be obtained using non-immune mouse IgM (not shown). St ganglioside standards. Solvent system: chloroform $/$ methanol $/ 0.2 \% \mathrm{CaCl}_{2}$, $50: 42: 11(\mathrm{v} / \mathrm{v})$

low); this was subcloned and selected for further study. The MAB produced was designated L9. The characterization of the other clones will be presented elsewhere.

$M A B L 9$ recognizes an acidic glycolipid. Acidic glycolipids extracted from the central and peripheral nervous systems of T. marmorata and rat were separated on TLC and immunostained with MAB L9. In the separated material from electric organ (Fig. 1, EO), an immunopositive band was

Fig. 2. Developmental expression of $L 9$ glycolipids. After immune staining, the chromatogram was sprayed with Ehrlich's reagent to render the total glycolipid pattern visible. St standard ganglioside; from the bottom: $G T 1 b$, fucosyl GD1b, GD1b, GD1a, fucosyl GM1, GM1, GM2, GM3. Solvent system as in Fig. 1 detected migrating between gangliosides GM1 and GD1a. An immunopositive band showing the same migration behaviour was also detected in separated glycolipid from electromotor nerves (EN) and brain (TB) of T. marmorata, and from the sciatic nerve (RS) of the adult rat. No staining was observed with material from adult rat brain (RB).

To investigate the chemical nature of the glycolipid antigen further, an acidic glycolipid fraction extracted from electric organ was extensively digested with neuraminidase and tested for immunostaining on TLC. This treatment affected neither the immunoreactivity of the antigen nor its $R_{F}$ value (result not shown). This shows that the glycolipid recognized by MAB L9 does not contain sialic acid and is not a ganglioside.

Developmental expression of the $L 9$ glycolipid. Although MAB L9 does not stain any acidic glycolipids in adult rat brain, immunoreactive bands are detected in glycolipid fractions extracted from embryonic rat brains between EDs 15 and 20 (Fig. 2). The epitope is first detected between EDs 13 and 15. At ED 15, two immunopositive bands migrating respectively between GM1 and GD1a and between GD1a and GD1b are detected. The expression of the epitope is maximal at ED 19 and thereafter declines during early post-natal life. At ED 19, a third immunoreactive band migrating in the lower region of the chromatogram appears. At post-natal day 20 , immunoreactivity is no longer detectable.

Subcellular distribution of the $L 9$ glycolipid. In order to obtain information concerning the subcellular distribution of

\section{MAB L9}

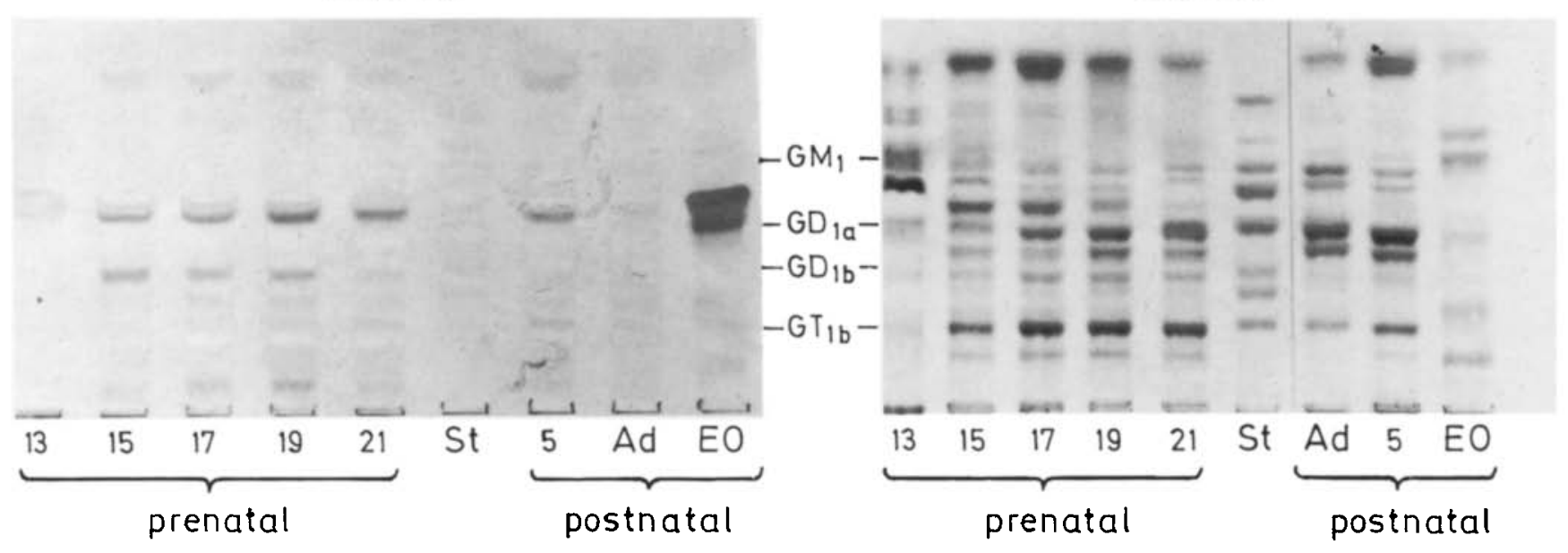



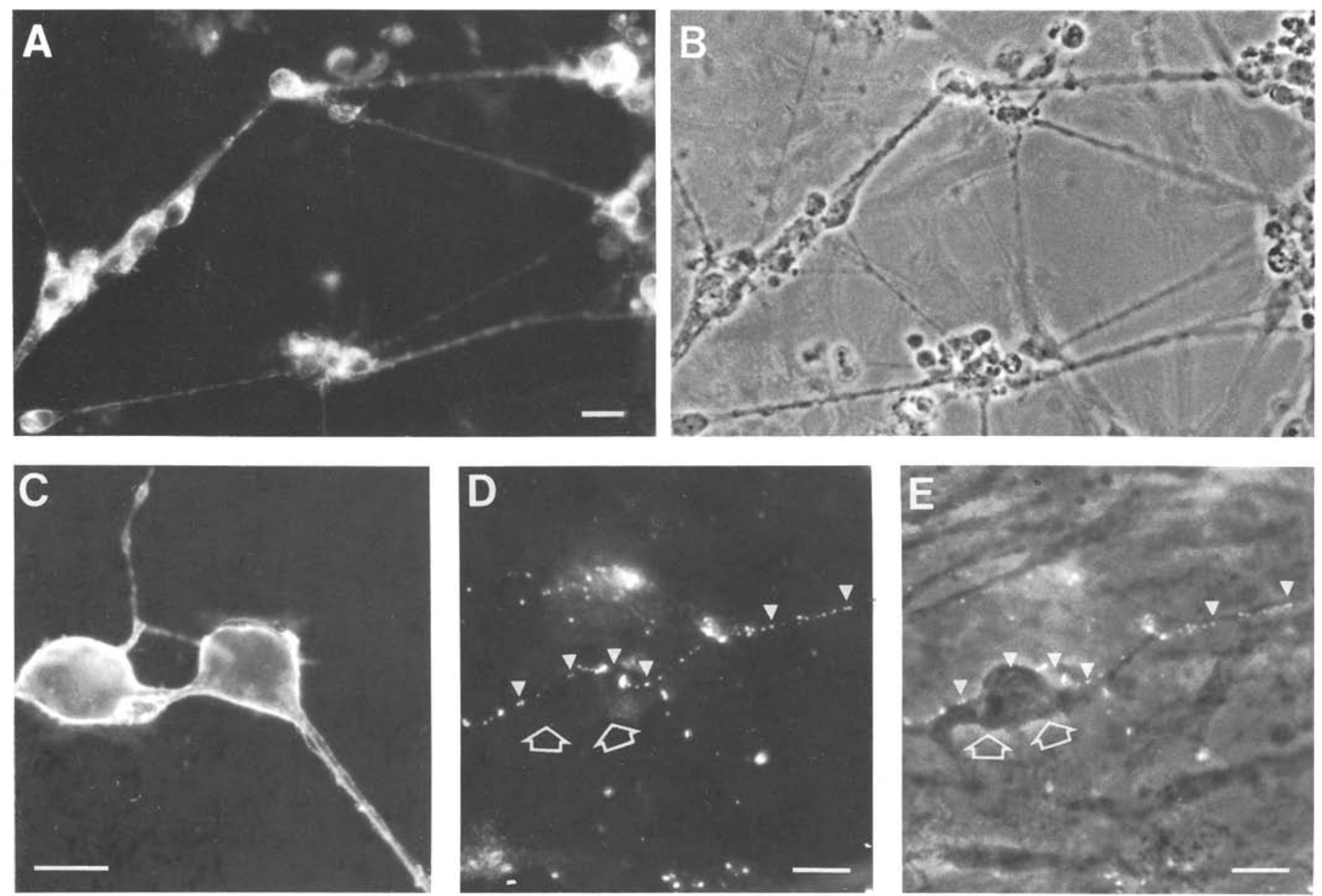

Fig. 3. Distribution of the L9 epitope in cell culture. Neural cells prepared from mouse spinal cord were kept in culture for 5 (A-C) and 10 days $(\mathbf{D}, \mathbf{E})$ and immune stained with MAB 19 . The membrane of cells displaying neuronal morphology was labelled on both cell body and processes (A and $\mathbf{C}$ ). B Phase contrast of $\mathbf{A}$. D Neuronal process immune stained by MAB L9; E Immunofluorescence + phase contrast of the same optical field as D. Punctated staining is distributed over a neuronal process (triangles) that run along an unstained cell body (empty arrows) and its processes. $\mathrm{A}, \mathrm{B} \times 320 ;$ bars $=20 \mu \mathrm{m} ; \mathrm{C} \times 1000 ;$ bar $=10 \mu \mathrm{m} ; \mathrm{D}, \mathrm{E} \times 800$; bars $=10 \mu \mathrm{m}$

the L9 antigen in developing rat brain, a fraction enriched in growth cones was isolated from the cortex of 4-day-old rats; its content of MAB L9-positive glycolipids was compared with that of a total membrane fraction from rats of the same age. The L9 antigen was found to be present in both growth cones and whole brain membranes, but, relative to ganglioside-bound sialic acid, there was no enrichment of the antigen in growth cones (data not shown).

\section{Distribution of the L9 epitope in cell culture}

To further characterize the cellular distribution of the L9 glycolipid, neural cells from embryonic spinal cord were cultured for different periods of time and stained with MAB L9. After 5 days in culture, a subpopulation of cells with neuronal features was stained with MAB L9 (Fig. 3A). Staining was distributed on the cell bodies and on the neu- ronal processes. Observations at higher magnification showed that the L9 epitope is primarily associated with the membranes of these cells (Fig. 3C). Some glial-like cells were also labelled, though with lower intensity. After 11 days of culture, long immunopositive processes presenting varicosity-like expansions (nodules) were observed (Fig. 3D, E). These processes were often seen to run along neuronal cell bodies and neuritic processes. Whether these varicosity-like expansions represent en-passant terminals making synapses is presently under investigation.

Since the L9 epitope is also present on various glycoproteins (see below), we have attempted to discriminate between glycolipidic and glycoproteic epitopes by pretreating the cells with chloroform/methanol prior to immune staining. This procedure is known to extract lipids and has been used by various authors as a diagnostic test for their presence (e.g., Richardson et al. 1982 and Scott et al. 1988). Chloroform/methanol pretreatment resulted in an overall reduction of the intensity of the staining, but no qualitative differences were observed (results not shown). Though this suggests that the immunoreactivity observed in all cell regions is due both to proteic and glycolipidic antigens, such an interpretation should be taken with caution since the chloroform/methanol treatment may potentially result in the denaturation of some of the proteic antigens.

\section{The relation between the $L 9$ and $H N K-1$ antigens}

The $L 9$ antigen is recognized by the $M A B, H N K-1$. It has recently been shown by Chou et al. (1986) that the MAB, 


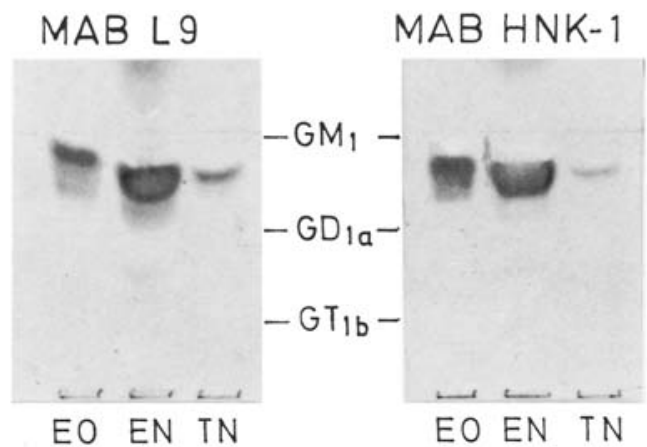

a

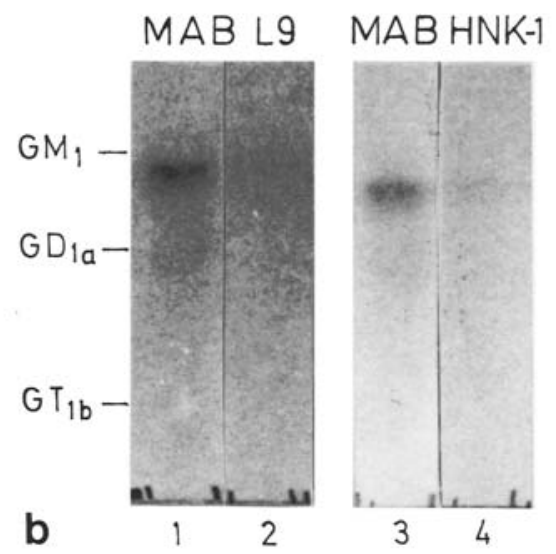

Fig. 4. Comparison of the immunoreactivity of MABs, $L 9$ and $H N K-1$. In (A), the reactivity of MABs, L9 and HNK-1, were tested against glycolipid extracted from electric organ $(E O)$, electric nerve $(E N)$ and $T$. marmorata brain $(T B)$. Both antibodies stain bands with identical $R_{F}$. In (B), a standard of purified sulphatylglucuronyl-paragloboside (lanes 1,3) and desulphated glucuronylparagloboside (lanes 2, 4) were immune stained with MABs, L9 (lanes 1, 2) and HNK-1 (lanes 3, 4). Both antibodies recognize the sulphoglycolipid but not its desulphated product, suggesting that the epitope recognized by the two MABs is related to the sulphoglucuronyl part of the molecule. The positions of the standard gangliosides are indicated

$\mathrm{HNK}-1$, originally raised against a membrane antigen from a human T-cell line, HSB-2 (Abo and Bach 1981; Abo et al. 1982), recognizes a glycolipidic antigen in extracts prepared from mammalian sciatic nerve; on TLC, this glycolipidic antigen has a mobility very similar to that of the L9 antigen. Noting this similarity, we have compared the reactivity of the $M A B, H N K-1$, with that of the MAB, L9, on Torpedo glycolipid. Figure 4A shows that MAB, HNK-1, indeed recognizes a glycolipid in our material from electric organ, electromotor nerves and $T$. marmorata brain with an $R_{F}$ indistinguishable from the $L 9$ antigen.

A sulphoglycolipid possesses an epitope common to the L9 and HNK-1 antigens. Chou et al. (1986b) have recently isolated from human peripheral nerves and cauda equina a sulphoglycolipid that is recognized by the MAB, HNK-1. It has the sequence Sulphate-3-GlcA $\beta(1 \rightarrow 3) \mathrm{Gal} \beta(1 \rightarrow$ 4) GlcNAc $\beta(1 \rightarrow 3) \mathrm{Gal} \beta(1 \rightarrow 4) \mathrm{Glc} \beta(1-1)$-ceramide and is thus identified as a sulphatylglucuronylparagloboside. Drs. Jungalwala and Chou have kindly tested the ability of our L9 MAB to recognize the purified sulphoglycolipid and have compared it with that of HNK-1. The results obtained are shown in Fig. $4 \mathrm{~B}$. Both MABs recognize the sulphoglycolipid but not its desulphated product; moreover, the $R_{F}$ value of the sulphoglycolipid is indistinguishable from the immunoreactive glycolipid in our extracts.

The L9 epitope is also expressed on proteic antigens. In the experiment presented in Fig. 5, homogenates prepared from 5-day-old and adult rat brain were submitted to polyacrylamide gel electrophoresis, blotted on nitrocellulose and finally reacted with the MABs, L9 and HNK-1. Both antibodies reacted with high molecular mass proteic antigens

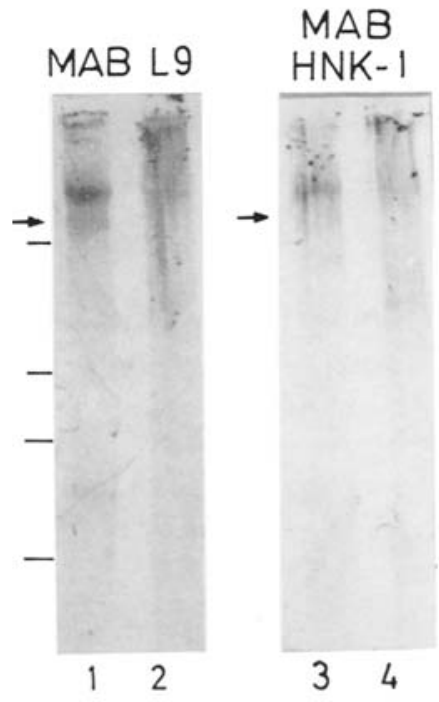

Fig. 5. Comparison of the reactivity of MABs, $L 9$ and $H N K-1$, against neural glycoproteins. Adult (lanes 1, 3) and 5-day-old (lanes 2, 4) rat brain homogenates were submitted to polyacrylamide gel electrophoresis. Proteins were then transferred to nitrocellulose and immune stained. Both MABs react with various highmolecular-mass antigens. The comparison of the two patterns of immunoreactivity shows that some of these antigens are recognized with higher sensitivity by the one or the other MAB (arrows). The position of the molecular-mass standards is indicated by adjacent lines; from the top: $200000,116000,96000$, and $66000 \mathrm{Da}$

in 5-day-old and adult rat brain. Thus, whereas the expression of the L9 epitope on brain glycolipid is limited to the embryonic and early post-natal life, glycoproteins bearing this epitope are also present in adult brain.

In addition, it should be noted that, although the general pattern of immunoreactivity of the two MABs is very similar, minor immunoreactive components appear to be recognized with higher sensitivity by one or the other $M A B$ (Fig. 5, arrows). This suggests a small difference in the affinity for the epitope recognized by the two MABs.

Immunohistochemical comparison of $M A B s, L 9$ and $H N K-1$. The immunoreactivity of the MABs, L9 and HNK-1, has also been compared in immunohistochemical studies on adult rat spinal cord (Fig. 6). The staining patterns are similar, but not identical. In the grey matter, the most prominent structures stained by the MAB, L9, are neuronal cell 

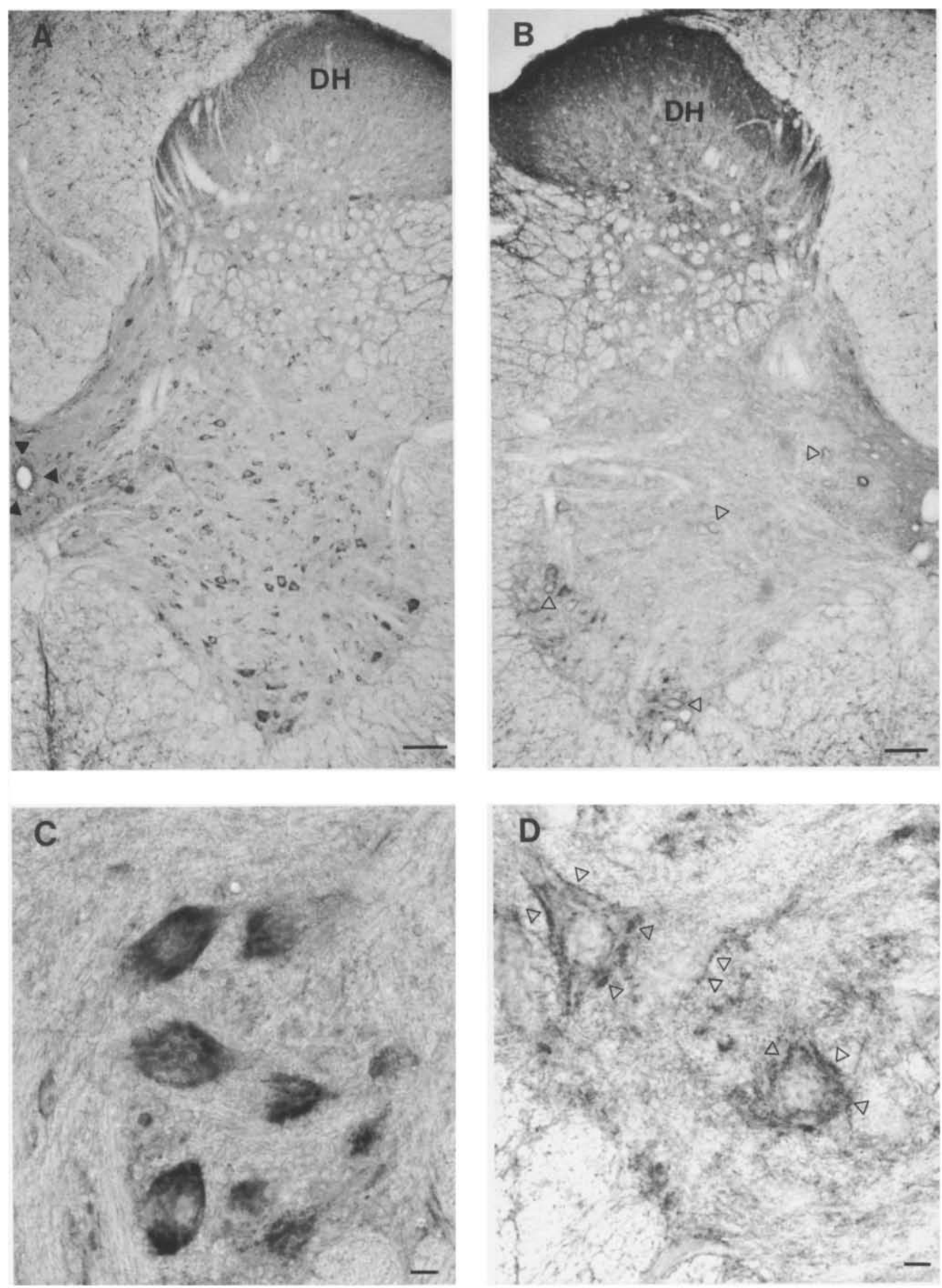
bodies (Fig. 6A, C). The staining is intense and appears to extend from the plasma membrane into the cytoplasm (Fig. 6C). Strong punctate staining is also observed in the dorsal horn in the region of the second lamina of Rexed. In the white matter, fibres are stained, together with the ependymal cells lining the central canal.

In contrast, fewer neurons are stained by anti-HNK-1 (Fig. 6B, D); the staining seems to be mainly distributed in a punctate manner on the plasma membrane with a pattern of staining that strongly resembles that obtained with antibodies directed against nerve terminal antigens. The region of the second lamina of Rexed is intensely stained, whereas, in contrast to MAB L9, the ependymal cells of the central canal are not stained.

\section{Discussion}

The MABs, L9 and HNK-1, may be regarded on the evidence presented here as recognizing identical or closely related epitopes. Both antibodies appear to recognize a sulphated carbohydrate sequence as shown by the fact that (a) they react with a purified sample of sulphated glucuronyl paragloboside, and (b) they are unable to recognize the desulphated glucuronyl paragloboside. In addition, both antibodies recognize acidic glycolipids with identical $\mathrm{R}_{\mathrm{F}}$ values, in Torpedo marmorata.

It is of interest that the two antibodies produce somewhat different staining patterns when compared immunohistochemically. This difference in staining is not necessarily inconsistent with the above-mentioned similarity of the two MABs, since it can be explained by differences in their affinities for the antigens and/or by slight differences in the epitopes recognized. The comparison of the immunoreactivity of the two MABs by Western blot experiments suggest that such factors are involved; although the general pattern of immunoreactivity appears to be very similar, some immunoreactive bands are stained with higher density by the one or the other MAB, suggesting differences in the affinity for the various antigens.

Because the L9/HNK-1 epitope is a carbohydrate and is carried by various molecules, it is likely that other parts of the 'host' molecule may influence the spatial conformation of the epitope itself. It is conceivable, therefore, that different MABs may recognize these epitope conformations with higher or lower affinity. This could account for the immunohistochemical difference in the distribution pattern of the immunoreactive component recognized.

The L9/HNK-1 epitope is also carried by a glycolipidic antigen expressed in the central and peripheral nervous system of $T$. marmorata during embryonic and adult life. In contrast, in rat brain, the expression of this epitope on

Fig. 6. Immunohistochemical comparison of MABs, L9 and HNK1. Rat spinal cord sections were stained with $(\mathbf{A}, \mathbf{C})$ MAB, L9, and (B, D) MAB, HNK-1. C, D Higher magnifications of comparable regions of the ventral horn. The MAB, L9, strongly stains neuronal cell bodies; staining is also observed in the dorsal horn $(D H)$ and on the ependymal cells (black triangles) of the central canal. With MAB, HNK-1, the highest degree of staining is observed in the dorsal horn $(D H)$. Fewer neurons are stained by this antibody; the staining seems to be mainly distributed as spots on the plasma membrane (empty triangles in B, D). A, B $\times 80$; bars $=100 \mu \mathrm{m} . \mathrm{C}, \mathrm{D} \times 500 ;$ bars $=10 \mu \mathrm{m}$ glycolipids is strictly developmentally regulated (Schwarting et al. 1987, and this paper). L9-positive glycolipids are first detected at ED 13, reaching their maximal expression at ED 19 and disappearing by post-natal day 20. Immunohistochemical and subcellular experiments suggest that these glycolipids are primarily located in the plasma membrane of neuronal cells undergoing differentiation.

It has recently been shown that glycolipids and tetrasaccharide carrying the HNK-1 epitope are able to interfere with cell-cell and cell-substrate interaction in vitro; this has led to the suggestion that the HNK-1 carbohydrate acts as a ligand in cell adhesion (Künemund et al. 1988). It should be noted, however, that whereas the glycoproteic epitope is expressed until adulthood, that of the glycolipidic epitope is restricted to a period in which important basic developmental processes take place in brain. Furthermore, in systems in which neuronal regeneration is known to occur in adult life, e.g. in fish brain and the mammalian peripheral nervous system, the L9 glycolipid is expressed throughout life; by contrast, in the mammalian central nervous system, where the ability to regenerate is lost early in development, the L9 glycolipid is not found in adult material. These glycolipids may thus play an important role in neural development and regeneration. The nature of this role merits further investigation, in which MABs of the L9 and HNK-1 type may well prove to be useful tools.

Acknowledgements. We are grateful to Drs. D.K.H. Chou and F.B. Jungalwala for their kind cooperation and to Dr. G.I. Kristiansson for preparing growth cones. The work was partly supported by the Deutsche Forschungsgemeinschaft (Grant No Wh 1/5-1 to V.P.W.) and by Fidia Pharmaforschung GmbH.

\section{References}

Abo T, Balch CM (1981) A differentiation antigen of human NK and $K$ cells identified by a monoclonal antibody (HNK-1). $J$ Immunol 127:1024-1029

Abo T, Cooper MD, Balch CM (1982) Characterization of Leu- $7^{+}$ (HNK-1) human lymphocytes, two distinct phenotypes of human NK cells with different cytotoxic capability. J Immunol 129:1752-1757

Bon S, Méflah K, Musset F, Grassi J, Massoulié J (1987) An immunoglobulin $\mathrm{M}$ monoclonal antibody recognizing a subset of acetylcholinesterase molecules from electric organs of Electrophorus and Torpedo, belongs to HNK-1 anti-carbohydrate family. J Neurochem 49:1720-1731

Borroni E, Derrington E (1987) A monoclonal antibody raised against glycolipids of Torpedo marmorata detects developmentally regulated glycolipids of mammalian brain. J Neurochem 48S: $84 \mathrm{~A}$

Borroni E, Derrington E, Kristjansson GI (1987) Identification of an evolutionarily conserved glycolipid and its developmental regulation in the rat central nervous system. Biol Chem HoppeSeyler 368:128

Brockaus M, Magnani JL, Blaszczyk SZ, Koprowski H, Karlsson K, Larson G, Ginsburg V (1981) Monoclonal antibodies directed against the human $\mathrm{Le}^{\mathrm{b}}$ blood group antigen. $\mathrm{J}$ Biol Chem 256:13223-13225

Chou DKH, Ilyas AA, Evans JE, Costello C, Quarles RH, Jungalwala FB (1986b) Structure of sulphated glucuronyl glycolipids in the nervous system reacting with HNK-1 antibody and some IgM paraproteins in neuropathy. J Biol Chem 261:1171711725

Derrington EA, Masco D, Whittaker VP (1988) Confirmation of the cholinergic specificity of the Chol-1 gangliosides in mammalian brain using affinity-purified antisera and lesions affecting the cholinergic input to the hippocampus. J Neurochem (in press) 
Ferretti P, Borroni E (1984) Effect of denervation on a cholinergicspecific ganglioside antigen (Chol-1) present in Torpedo electromotor presynaptic plasma membranes. J Neurochem 42: 1085-1093

Ferretti P, Borroni E (1986) Putative cholinergic-specific gangliosides in guinea-pig forebrain. J Neurochem 46:1888-1894

Fiedler W, Borroni E, Ferretti P (1986) An immunochemical study of synaptogenesis in the electric organ of Torpedo marmorata by use of antisera to vesicular and presynaptic plasma membrane components. Cell Tissue Res 246:439-445

Galanos C, Lüderitz O, Westphal O (1971) Preparation and properties of antisera against the lipid-A component of bacterial lipopolysaccharides. Eur J Biochem 24:116-122

Gordon-Weeks PR, Lockerbie RO (1984) Isolation and partial characterization of neuronal growth cones from neonatal rat forebrain. Neuroscience 13:119-136

Grumet M, Hoffman S, Crossin KL, Edelman G (1985) Cytotactin, an extracellular matrix protein of neuronal and non-neuronal tissue mediates glia-neuron interaction. Proc Natl Acad Sci USA 82: 8075-8077

Ilyas AA, Quarles RH, Brady RO (1984a) The monoclonal antibody HNK-1 reacts with a human peripheral nerve ganglioside. Biochem Biophys Res Comm 122:1206-1211

Ilyas AA, Quarles RH, MacIntosh TD, Dobersen MJ, Trapp BD, Dalakas MC, Brady RO (1984b) IgM in a human neuropathy related to paraproteinemia binds to a carbohydrate determinant in the myelin-associated glycoprotein and to a ganglioside. Proc Natl Acad Sci USA 81:1225-1229

Kruse J, Mailhammer R, Wernecke H, Faissner A, Sommer I, Goridis C, Schachner M (1984) Neural cell adhesion molecule and myelin-associated glycoprotein share a common carbohydrate moiety recognized by monoclonal antibody L 2 and HNK1. Nature (Lond) 311:153-155

Kruse J, Keilhauer G, Gaissner A, Timpl R, Schachner M (1985)
The $\mathrm{J} 1$ glycoprotein - a novel nervous system cell adhesion molecule of the L2/HNK-1 family. Nature (Lond) 316:146-148

Künemund V, Jungalwala FB, Fisher G, Chou DKH, Keilhauer G, Schachner M (1988) The L2/HNK-1 carbohydrate of neural cell adhesion molecules is involved in cell interactions. J Cell Biol 106:213-223

Poltorak MR, Sadoul R, Keilhauer G, Landa C, Schachner M (1987) The myelin-associated glycoprotein (MAG), a member of the L2/HNK-1 family of neural cell adhesion molecules, is involved in neuron-oligodendrocyte and oligodendrocyte-oligodendrocyte interaction. J Cell Biol 105:1893-1899

Rathjen FG, Schachner M (1984) Immunocytological and biochemical characterization of a new neuronal cell surface component (L1 antigen) which is involved in cell adhesion. EMBO J $3: 1-10$

Richardson PJ, Walker JH, Jones RT, Whittaker VP (1982) Identification of a cholinergic-specific antigen Chol-1 as a ganglioside. J Neurochem 38:1605-1614

Schwarting GA, Jungalwala FB, Chou DKH, Boyer AM, Yamamoto $M$ (1987) Sulphated glucuronic acid-containing glycoconjugates are temporally and spatially regulated antigens in the developing mammalian nervous system. Dev Biol 120:65-76

Scott JCL, Bacon F, Sanes JR (1988) A synapse-specific carbohydrate at the neuromuscular junction: association with both acetylcholinesterase and a glycolipid. J Neurosci 8:932-944

Shashoua VE, Daniel PF, Moore ME, Jungalwala FB (1986) Demonstration of glucuronic acid on brain glycoproteins which react with HNK-1 antibody. Biochem Biophys Res Comm 138:902-909

Tettamanti G, Bonali F, Marchesini S, Zambotti V (1973) A new procedure for the extraction, purification and fractionation of brain gangliosides. Biochim Biophys Acta 296:160-170

Accepted December 21, 1988 